\title{
Practical Distribution State Estimation Using Hybrid Particle Swarm Optimization
}

\author{
Shigenori Naka \\ Takamu Genji, Toshiki Yura \\ Technical Research Center, \\ Kansai Electric Power Co., Inc. \\ 3-11-20, Nakoji, Amagasaki, Hyogo, 661 Japan
}

\author{
Yoshikazu Fukuyama, member, IEEE \\ Power Technology Laboratory \\ Fuji Electric Corporate R \& D, Ltd. \\ No. 1, Fuji-machi, Hino-city, Tokyo, 191 Japan
}

\begin{abstract}
This paper proposes a practical distribution state estimation method using a hybrid particle swarm optimization. The proposed method considers nonlinear characteristics of the practical equipment and actual measurements in distribution systems. The method can estimate load and distributed generation output values at each node by minimizing difference between measured and calculated state variables. The feasibility of the proposed method is demonstrated and compared with the original PSO on practical distribution system models. The results indicate the applicability of the proposed state estimation method to the practical distribution systems.
\end{abstract}

Keywords: State Estimation, Distribution Systems, Modern Heuristic Method, Hybrid Particle Swarm Optimization, Distributed Generation, Voltage Regulator

\section{INTRODUCTION}

On-line state estimation is becoming one of the key functions in distribution control centers considering deregulation environment and introduction of distributed generator (DG) in distribution systems. Distribution state estimation (DSE) is required to consider error and asynchronism of measurement from actual distribution systems. Since limited measurement values are obtained from actual distribution systems, DSE has to realize high accuracy estimation with limited measurement.

DSE is usually formulated as a weighted least mean square (WLMS) problem. Equipment in distribution systems such as SVC and DG causes nonlinear characteristics of the objective function for DSE. For example, static var compensators (SVCs ) have nonlinear output characteristics. Transformers with automatic tap changer have a discrete tap control function. Therefore, target load flow equations may be changed because of automatic tap changing function of voltage controllers during convergence. Output characteristics of induction generators can be described by a nonlinear function expressed by constant impedance, constant current, and constant power (ZIP) load. A number of methods have been developed as an advanced function of distribution control centers [1-11]. The methods can be divided into categories: statistical and load adjustment SE formulation. The former methods usually utilize an iterative convergence method such as Quasi-Newton method and the latter methods usually utilize sensitivity analysis. Since conventional distribution state estimation methods belonging to both categories assume that the objective function or equations related to DSE can be differentiable and continuous. However, considering the above-mentioned nonlinear characteristics of the practical equipment in distribution systems, the objective function and the equations can not be differentiable and continuous, and the conventional methods can not be applied. Therefore, A practical distribution state estimation method has been eagerly awaited considering the practical requirements.

Modern heuristic algorithms are considered as practical tools for nonlinear optimization problems [12]. The algorithms do not require that the objective function has to be differentiable and continuous. A particle swarm optimization (PSO) is one of the modern heuristic algorithms [13][14] and can be applied to continuous nonlinear optimization problems such as DSE. It has been developed through simulation of simplified social models. A hybrid PSO (HPSO) adds a selection mechanism of the evolutionary computation (EC) to PSO and it can generate high quality solution within short calculation time [15]. Since the state estimation is one of the on-line functions in distribution control centers, HPSO must be an appropriate method for the target problem.

This paper proposes a practical distribution state estimation method using a hybrid particle swarm optimization. The proposed method considers practical measurements in actual distribution systems and assumes that absolute values of voltage and current can be measured at the secondary side buses of S/Ss and RTUs in distribution systems. The method can handle nonlinear characteristics of the practical equipment in distribution systems. It can estimate load and distributed generation output values at each node by minimizing difference between measured and calculated state variables. The feasibility of the proposed method is demonstrated and compared with the original PSO on practical distribution system models.

\section{FORMULATION OF DISTRIBUTION STATE ESTIMATION}

\section{Measurement data and assumptions}

The following data are assumed to be obtained from actual 
distribution networks:

(1) Substation: absolute value of sending voltage and current,

(2) RTU: absolute value of voltage and current.

In addition, the following assumptions are required for the state estimation considering the above limited measured data:

(1) A contracted load value is known at each load section.

(2) Estimated power factor of sending end at S/S and each section can be obtained.

(3) If output of DG is fixed, the output and power factor of DG can be obtained. If output of DG is variable, the estimated output and power factor of DG can be obtained.

Fig. 1 shows the measured data.

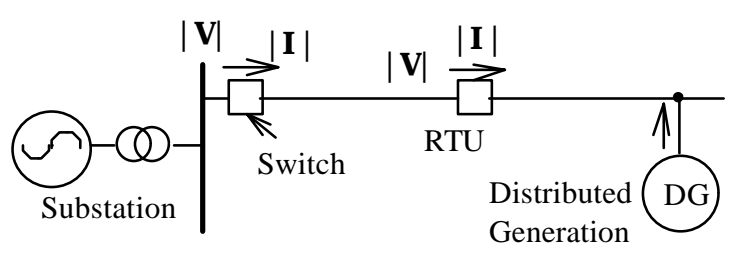

Fig.1 Measured Data.

\section{Formulation}

The objective function of the distribution state estimation is the same as that of conventional state estimation as follows:

$$
\min J(x)=\sum_{i=1}^{m} w_{i}\left(z_{i}-h_{i}(x)\right)^{2}
$$

where, $\mathrm{x}$ : state variable, $\mathrm{w}_{\mathrm{i}}$ : weighting factor of measurement variable $\mathrm{i}$, $\mathrm{z}_{\mathrm{i}}$ : measurement value of measurement variable $\mathrm{i}$, $h_{i}$ : state equation of measurement variable $i$.

Namely, the function is to minimize the difference between measured and calculated state variables. It should be noted that one of the state variables is a load value at each section rather than voltage as utilized by the conventional state estimation. Load power factor is assumed to be fixed as mentioned above. Therefore, only an active power load value is utilized as a state variable. The output value of DG is also utilized as a state variable. The state variables are calculated among the following bounds. The center value of the bound at each load is calculated using the total input power to the target network and the ratio of the target load section to the total loads of the target network. The center value of the bound of each variable output DG is average output of the DG.

$$
x_{j, \min } \leq x_{j} \leq x_{j, \max }
$$

where, $\mathrm{x}_{\mathrm{j}, \text { min }}$ : minimum value of state variable $\mathrm{j}$,

$\mathrm{x}_{\mathrm{j}, \max }$ : maximum value of state variable $\mathrm{j}$.

Voltage and current can be calculated by fast distribution power flow (backward forward sweep (BFS) method) [16]. Consequently, the state estimation problem can be formulated as a constrained nonlinear optimization problem. Considering the nonlinear characteristic of actual equipment in distribution system, conventional nonlinear optimization methods based on nonlinear programming techniques can not be applied and HPSO should be utilized as an optimization method as mentioned below.

\section{HYBRID PARTICLE SWARM OPTIMIZATION}

\section{Particle Swarm Optimization [13][14]}

PSO is one of the optimization techniques and belongs to EC techniques. The method has been developed through a simulation of simplified social models. The features of the method are as follows:

(1) The method is based on researches on swarms such as fish schooling and bird flocking.

(2) It is based on a simple concept. Therefore, the computation time is short and it requires few memories.

According to the research results for bird flocking, birds are finding food by flocking (not by each individual). It leaded the assumption that information is owned jointly in flocking. According to observation of behavior of human groups, behavior pattern of each individual is based on several behavior patterns authorized by the groups such as customs and the experiences by each individual (agent). The assumptions are basic concepts of PSO.

PSO is basically developed through simulation of bird flocking in two-dimension space. The position of each individual (agent) is represented by $\mathrm{XY}$ axis position and also the velocity is expressed by $v x$ (the velocity of $X$ axis) and vy (the velocity of $\mathrm{Y}$ axis). Modification of the agent position is realized by the position and velocity information.

An Optimization technique based on the above concept can be described as follows: namely, bird flocking optimizes a certain objective function. Each agent knows its best value so far (pbest) and its XY position. Moreover, each agent knows the best value so far in the group (gbest) among pbests. Each agent tries to modify its position using the following information:

- the current positions $(\mathrm{x}, \mathrm{y})$,

- the current velocities (vx, vy),

- the distance between the current position, and pbest and gbest.

This modification can be represented by the concept of velocity. Velocity of each agent can be modified by the following equation:

$$
v_{i}^{k+1}=w v_{i}^{k}+c_{1} \text { rand } \times\left(\text { pbest }_{i}-s_{i}^{k}\right)+c_{2} \text { rand } \times\left(\text { gbest }-s_{i}^{k}\right)
$$

$$
\begin{aligned}
\text { where, } \mathrm{v}_{\mathrm{i}}{ }^{\mathrm{k}} & \text { : velocity of agent } \mathrm{i} \text { at iteration } \mathrm{k}, \\
\mathrm{w} & : \text { weighting function, } \\
\mathrm{c}_{\mathrm{j}} & : \text { weighting factor, } \\
\text { rand }^{\mathrm{k}} & : \text { random number between } 0 \text { and } 1, \\
\mathrm{~s}_{\mathrm{i}} & : \text { current position of agent } \mathrm{i} \text { at iteration } \mathrm{k}, \\
\text { pbest }_{\mathrm{i}} & : \text { pbest of agent } \mathrm{i}, \\
\text { gbest } & : \text { gbest of the group. }
\end{aligned}
$$


Using the above equation, a certain velocity which gradually gets close to pbest and gbest can be calculated. The current position (searching point in the solution space) can be modified by the following equation:

$$
s_{i}^{k+1}=s_{i}^{k}+v_{i}^{k+1}
$$

Fig. 2 shows a concept of modification of a searching point by PSO and Fig. 3 shows a searching concept with agents in a solution space.

\section{Hybrid Particle Swarm Optimization [15]}

HPSO utilizes the mechanism of PSO and the natural selection mechanism which is usually utilized by EC such as genetic algorithms (GAs). Since search procedure by PSO deeply depends on pbest and gbest, the searching area is limited by pbest and gbest. On the contrary, by introduction of the natural selection mechanism, effect of pbest and gbest is gradually vanished by the selection and broader area search can be realized. Agent positions with low evaluation values are replaced by those with high evaluation values using the selection. On the contrary, pbest information of each agent is maintained. Therefore, intensive search in a current effective area and dependence on the past high evaluation position are realized. Fig. 4 shows a general flow chart of HPSO. Fig. 5 shows concept of step. 2, 3, and 4 of the flow chart.

\section{DISTRIBUTION STATE ESTIMATION BY HPSO}

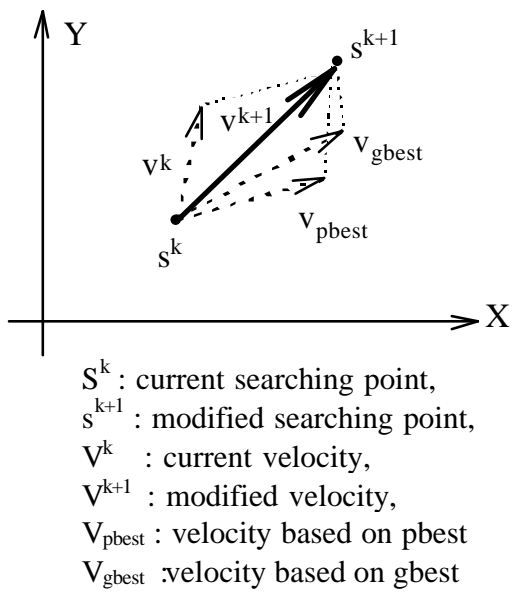

Fig. 2 Concept of modification of a searching point by PSO.

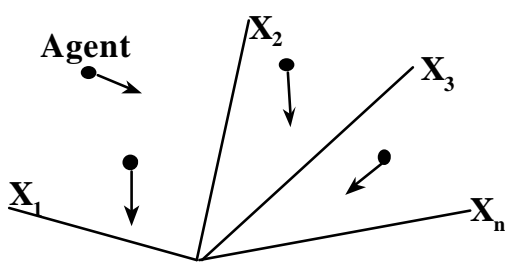

Fig. 3 Searching concept with agents in a solution space by PSO.

\section{State Variables}

DG output and load values are considered to be state variables as mentioned above. The variables can be calculated as follows in HPSO algorithm:

(1) DG output

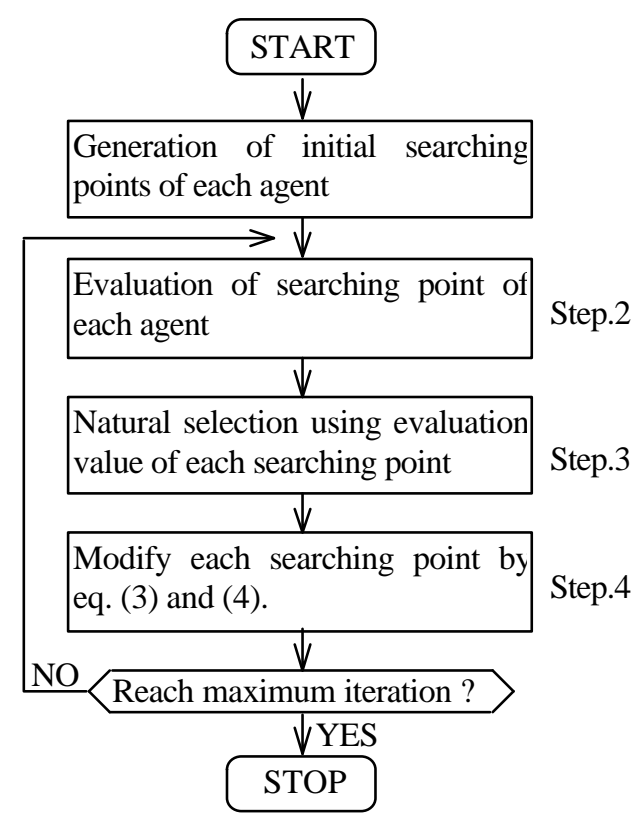

Fig.4 A general flow chart of HPSO.
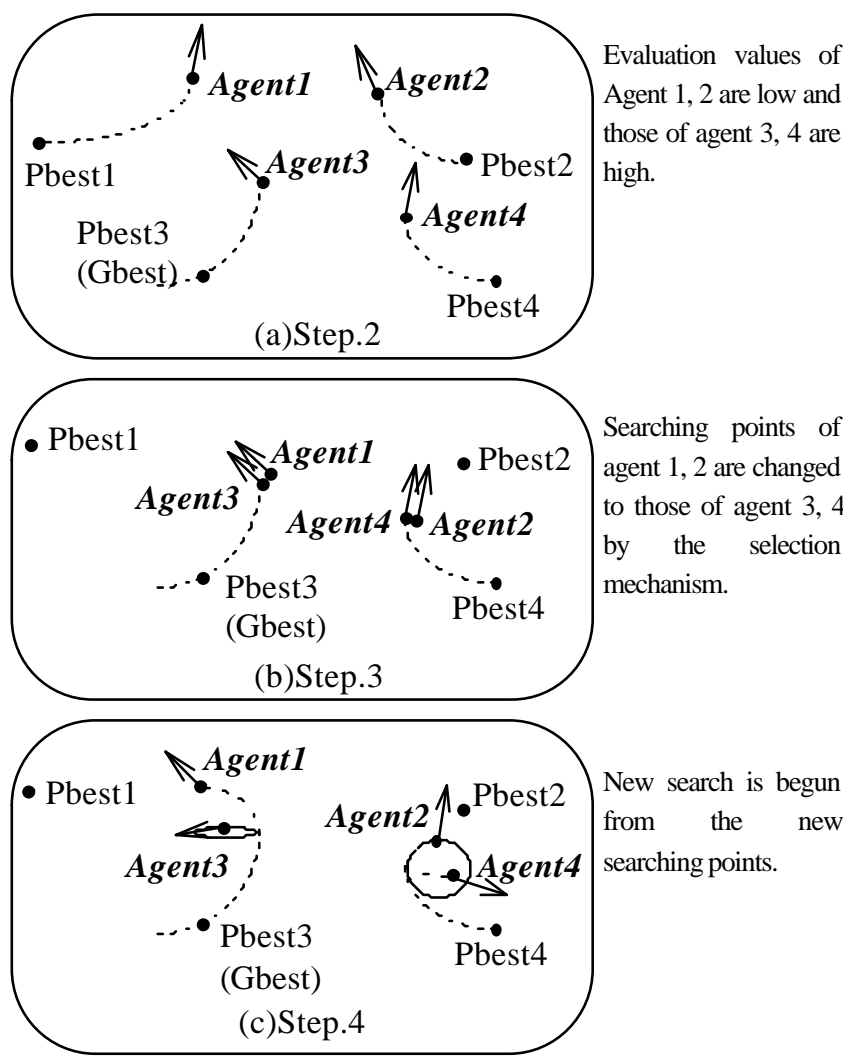

Fig. 5 Concept of searching process by HPSO. 


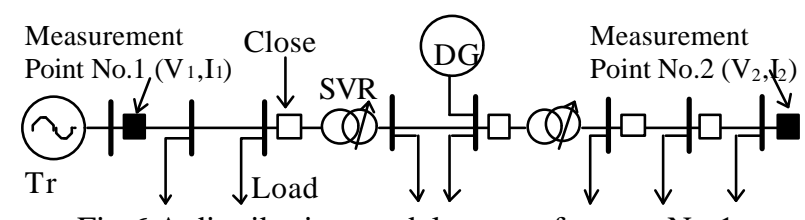

Fig.6 A distribution model system for case No.1.

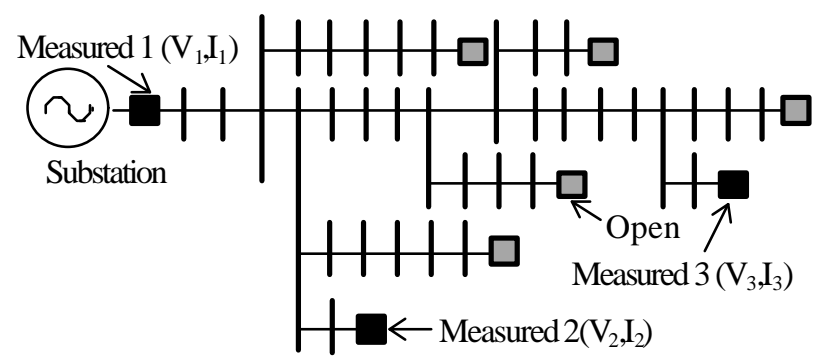

Fig.7 A distribution model for case No.2.

If the output of a DG is fixed, it is not utilized as a state variable and can be utilized as a specified value in load flow calculation. On the contrary, if the output of a DG is variable, the average and upper and lower limits of the output is set considering the target power system conditions.

An initial value of DG output can be calculated between upper and lower limits of the output at each agent. The state variables can be modified between the limits in search procedures.

\section{(2) Load values}

Average load value at each load section can be caluculated with the ratio of the contracted section load value to the total contracted load values, and total injection power to the target power system. Upper and lower limits of the load values can be calculated considering heavy and light loading conditions of the target power system.

An initial value of the load can be calculated between upper and lower limits of the load value at each agent. The state variables can be modified between the limits in search procedures.

\section{The proposed algorithm}

The following algorithm is utilized for the state estimation:

Step 1 Input data

The following data are input.

- network configuration, line impedance

- contracted load value

- measurement data (S/S, RTU, and DG)

Step 2 Set calculation conditions

(1) Calculation of initial values of state variables

- Using total power input to the target network and ratio of the contracted load value of each load section to the total contracted load values of the target network, initial value of each load is calculated.

- Using average power output of each DG, initial value of each DG is calculated.

Using initial values of state variables, initial load flow calculation by BFS is performed.

(2) Set upper and lower bounds of state variables

- Using the results of initial load flow calculation, upper and

lower bounds of each state variable can be calculated.

Step 3 State estimation

Find network condition which minimizes error between measurement values and calculated values by HPSO.

The proposed distribution state estimation handles constrained nonlinear optimization problem expressed by eq. (1) and (2). Generally, it is difficult to give a suboptimal initial solution to the optimization problem. However, using the problem-dependent knowledge, initial load and DG output values can be given for the taget problem as shown before. Therefore, it can be estimated that the proposed method has a good convergence characteristic.

Practically, it is necessary to satisfy limited number of relations between measured and calculated values. Therefore, there may exit many solutions which can satisfy the constrains. HPSO has a global optimization characteristic and can generate consistence load and DG output values, which can minimize the objective function without falling into local minima. Therefore, if upper and lower limits of state variables are set to impractical values, the variables may be converged to impractical values. Namely, impractical values of state variables can minimize the objective function more than practical values. However, there exists only a unique solution for radial network load flow, while loop networks have mutiple solutions [17]. Therefore, the algorithm can generate practical state variable values if measured values are reasonable. According to authors' expriences, solutions have always converged to appropriate values.

Solutions can be maintained within reasonable values by restriction of upper and lower limits of state variables. If solutions can not be obtained within reasonable limits, the limits can be expanded. Then, if an impractical solution is obtained, it can be predicted that bad data are included in measured data. Namely, using the expansion, detection of bad data can be realized. The detection of bad data can be considered in step 3 of the above-mentioned proposed algorithm.

\section{V.NUMERICAL EXAMPLES}

\section{Simulation Conditions}

The proposed method and a method based on the conventional PSO are applied to distribution model systems. The followings are test cases:

(1) Case 1

The methods are applied to a model system as shown in fig. 6 which models rural area. Load flow calculation results are utilized as measured data. Namely, capability of the methods converging to the values near to the measured data are 
compared. The model has one DG and two voltage regulators (SVR). SVR is widely utilized in Japan and it automatically changes tap position of the transformer to regulate the voltage at a target point in distribution systems. These equipment causes nonlinear characteristics of the objective function.

(2) Case 2

The methods are applied to a model system as shown in fig. 7, which models urban area with actually measured data. Namely, Effectiveness of the methods considering measured error is evaluated.

Weighting coefficients of eq. (1) are set to 1.0. The weighting function of eq. (3) is set to the following equation :

$$
w=w_{\max }-\frac{w_{\max }-w_{\min }}{\text { iter }_{\max }} \times \text { iter }
$$

where, $\mathrm{w}_{\max }$ : Initial value of weighting coefficient,

$\mathrm{w}_{\min }$ : Final value of weighting coefficient,

iter $_{\max }$ : Maximum iteration,

iter : Current iteration number.

The number of agent is set to 20. 30 trials are performed for appropriate parameter determination simulation and 100 trials are performed for other simulations using different random numbers. At each trial, the best-evaluated value is stored within 100 searching iteration.

\section{Simulation Results}

\section{(1) Case 1}

HPSO have parameters: $\mathrm{w}_{\max }, \mathrm{w}_{\min }$, and $\mathrm{C}_{\mathrm{i}}$ in eq. (3). Table 1 shows average values of objective function values for various parameter combinations. According to the results, appropriate combination of $\mathrm{w}_{\max }$ and $\mathrm{w}_{\min }$ is 0.9 and 0.4 , and Appropriate $\mathrm{C}_{\mathrm{i}}$ is 1.0 to 2.0. The values are the same as those recommended by other papers [13][14]. The following values are appropriate for DSE and utilized for other simulations: $\mathrm{w}_{\max }=0.9, \mathrm{w}_{\min }=0.4$, $C_{i}=2.0$. Namely, the appropriate parameter values are not different among various target problems. This is one of the desirable characteristics of PSO.

State estimation results are shown in Table 2, 3 and fig. 6. As shown in Table 2, the minimum evaluation values by HPSO and PSO are the same. However, the maximum evaluation value, which has a maximum error between measurement data

Table 1 Average values of objective function values to various parameter combinations (case No.1).

\begin{tabular}{|c|c|c|c|}
\hline & \multicolumn{3}{|c|}{$\mathrm{W}_{\max }, \mathrm{W}_{\min }$} \\
\hline $\mathrm{c}_{. \%}$ & \multicolumn{3}{|c|}{} \\
\hline & $0.9,0.4$ & $2.0,0.9$ & $2.0,0.4$ \\
\hline 0.5 & 0.000031 & 0.000588 & 0.000119 \\
\hline 1.0 & $\mathbf{0 . 0 0 0 0 2 0}$ & 0.000332 & 0.000112 \\
\hline 1.5 & $\mathbf{0 . 0 0 0 0 1 8}$ & 0.000199 & 0.000157 \\
\hline 2.0 & $\mathbf{0 . 0 0 0 0 1 9}$ & 0.000165 & 0.000106 \\
\hline 2.5 & 0.000028 & 0.000126 & 0.000129 \\
\hline 3.0 & 0.000024 & 0.000152 & 0.000120 \\
\hline 4.0 & 0.000039 & 0.000085 & 0.000129 \\
\hline
\end{tabular}

and calculated value, by HPSO is approximately $59 \%$ of that by PSO and average evaluation value by HPSO is approximately $50 \%$ of that by PSO. The results indicate HPSO can generate higher quality solutions by PSO without measurement errors. Table 3 and fig. 8 indicates the high quality solutions by HPSO as well.

\section{(2) Case 2}

Table 4 shows the estimation results for the model system in fig. 5. The proposed method gradually decreases load values in order to match it with the sending current value. As a result, calculated voltage values are higher than the measured values. The results are obtained considering

Table 2. Comparison of objective function values by both methods.

\begin{tabular}{c|c|c|c}
\hline Methods & Max. & Min. & Ave. \\
\hline HPSO & 0.000106 & 0.000000 & 0.000015 \\
\hline PSO & 0.000180 & 0.000000 & 0.000030 \\
\hline
\end{tabular}

Table 3. Comparison of measured and estimated voltage and current (case No.1).

\begin{tabular}{c|c|c|c|c}
\hline $\begin{array}{l}\text { Measur- } \\
\text { ed Point }\end{array}$ & $\begin{array}{l}\text { Measured } \\
\text { value }\end{array}$ & \multicolumn{2}{|c|}{ HPSO } & PSO \\
\hline & & $\begin{array}{l}\text { Optimal } \\
\text { estimation } \\
\text { value }\end{array}$ & $\begin{array}{l}\text { Ave. } \\
\text { estimation } \\
\text { value }\end{array}$ & $\begin{array}{l}\text { Ave. } \\
\text { estimation } \\
\text { value }\end{array}$ \\
\hline $\mathrm{I}_{1}$ & $150.00[\mathrm{~A}]$ & $150.01[\mathrm{~A}]$ & $149.98[\mathrm{~A}]$ & $150.22[\mathrm{~A}]$ \\
\hline $\mathrm{V}_{2}$ & $6500[\mathrm{~V}]$ & $6503[\mathrm{~V}]$ & $6481[\mathrm{~V}]$ & $6538[\mathrm{~V}]$ \\
\hline $\mathrm{I}_{2}$ & $0.00[\mathrm{~A}]$ & $0.00[\mathrm{~A}]$ & $0.00[\mathrm{~A}]$ & $0.00[\mathrm{~A}]$ \\
\hline
\end{tabular}

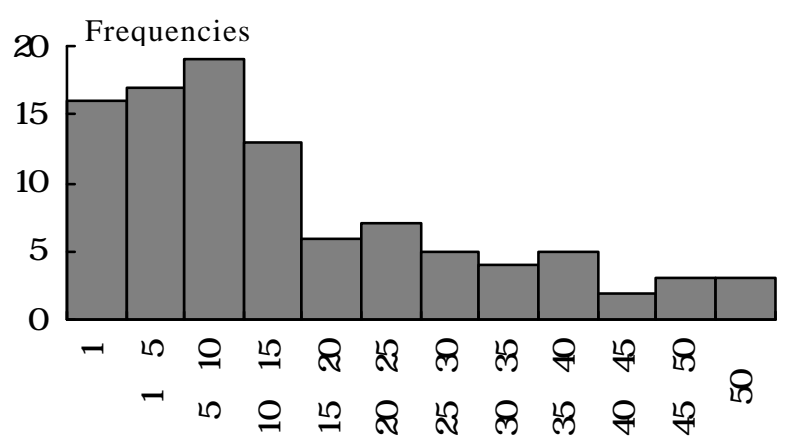

Evaluation interval $[\times 1$. Oe- 6$]$

Fig.8 Convergence characteristics of the proposed method (case No.1, 100 trials).

Table 4. Comparison of measured and estimated voltage and current for case No. 2 by HPSO.

\begin{tabular}{c|c|c}
\hline Target & $\begin{array}{c}\text { Measured } \\
\text { values }\end{array}$ & Optimal estimated values \\
\hline $\mathrm{I}_{1}$ & $320.00[\mathrm{~A}]$ & $320.03[\mathrm{~A}]$ \\
\hline $\mathrm{V}_{2}$ & $6705[\mathrm{~V}]$ & $6744[\mathrm{~V}]$ \\
\hline $\mathrm{V}_{3}$ & $6675[\mathrm{~V}]$ & $6706[\mathrm{~V}]$ \\
\hline
\end{tabular}


Table 5. Comparison of number of calculation by HPSO and PSO.

\begin{tabular}{c|c|c|c|c}
\hline & \multicolumn{2}{|c|}{ Case No.1 } & \multicolumn{2}{c}{ Case No.2 } \\
\hline & HPSO & PSO & HPSO & PSO \\
\hline \# of Nodes & \multicolumn{2}{|c|}{20} & \multicolumn{2}{c}{41} \\
\hline $\begin{array}{c}\text { \# of } \\
\text { Measure- } \\
\text { ment }\end{array}$ & \multicolumn{2}{|c|}{4} & \multicolumn{2}{c}{6} \\
\hline Flops & 12834882 & 12328668 & 14550867 & 14543764 \\
\hline
\end{tabular}

*)Flops means the calculation number counted in Matlab.

measurement errors and the appropriate results are obtained. Table 5 shows comparison of the number of calculations (Flops by Matlab) by both methods. The number by HPSO is almost same as that by PSO. Namely, the results indicate possibility of HPSO to generate high quality solutions compared with the original PSO.

\section{CONCLUSIONS}

This paper proposes a dis tribution state estimation method using a hybrid particle swarm optimization. The results of the paper can be summarized as follows:

(1) This paper develops a hybrid particle swarm optimization which can handle the non-differential and non-continuous objective function of distribution state estimation caused by nonlinear characteristics of the practical equipment such as SVC, SVR.

(2) The proposed method can estimate load and distributed generation output values at each node with practical limited measurement values in distribution systems.

(3) The results of the numerical simulations indicate that the proposed method can estimate the target system conditions more accurate than the original PSO. Moreover, it can estimate the appropriate target system conditions with measurement errors.

\section{REFERENCES}

[1] F. F. Wu and A. F. Neyer, "Asynchronous Distributed State Estimation for Power Distribution Systems", Proc. of 10th PSCC, Aug. 1990.

[2] I. Roytelman and S. M. Shahidehpour, "State Estimation for Electric Power Distribution Systems in Quasi Real-time Conditions", IEEE Trans. on Power Delivery, Vol. 8, No. 4, October 1993.

[3] M. E. Baran and A. W. Kelley, "State Estimation for Real-time Monitoring of Distribution Systems", IEEE Trans. on Power Systems, Vol. 9, No. 3, August 1994.

[4] M. E. Baran, A. W. Kelley, "A Branch-Current-Based State Estimation Method for Distribution Systems", IEEE Trans. on Power Systems, Vol. 10, No. 1, February 1995.

[5] C. N. Lu, J. H. Teng, and W. E. Liu, "Distribution System State Estimation", IEEE Trans. on Power Systems, Vol. 10, No. 1, February 1995.

[6] W. M. Lin, J. H. Teng, "State Estimation for Distribution Systems with Zero-Injection Constraints", IEEE Trans. on Power Systems, Vol. 11, No. 1, February 1996.

[7] Ke Li, "State Estimation for Power Distribution System and
Measurement Impacts", IEEE Trans. on Power Systems, Vol. 11, No. 2, May 1996.

[8] A. P. S. Meliopoulos, F. Zhang, "Multiphase Power Flow and State Estimation for Power Distribution Systems", IEEE Trans. on Power Systems, Vol. 11, No. 2, May 1996.

[9] R. C. Leou, C. N, Lu, National Sun Yat-Sen University, Taiwan, "Improving Feeder Voltage Calculation Results with Telemetered Data", IEEE Trans. on Power Delivery, Vol.11, No.4, October 1996.

[10] A. K. Ghosh, D. L. Lubkeman, M. J. Downey, and R. H. Jones, "Distribution Circuit State Estimation Using a Probabilistic Approach", IEEE Trans. on Power Systems, Vol. 12, No. 1, February 1997.

[11] W. H. E. Liu, et al., "A Practical State Estimation Algorithm for Distribution Management Systems", Proc. of $13^{\text {th }}$ PSCC, June 1999.

[12] C. R. Reeves, Modern Heuristic Techniques for Combinatorial Problems, Blackwell Scientific Publications, 1993.

[13] J. Kennedy and R. Eberhart, "Particle Swarm Optimization", Proc. of IEEE International Conference on Neural Networks (ICNN), Vol.IV, pp.1942-1948, Perth, Australia, 1995.

[14] Y. Fukuyama, et al., "A Particle Swarm Optimization for Reactive Power and Voltage Control Considering Voltage Stability", Proc. of IEEE International Conference on Intelligent System Application to Power Systems (ISAP), Rio de Janeiro, April 1999.

[15] P. Angeline, "Using Selection to Improve Particle Swarm Optimization", Proc. of IEEE International Conference on Evolutionary Computation (ICEC), Anchorage, May 1998.

[16] Y. Fukuyama, et al., "Parallel Power Flow Calculation in Electric Distribution Networks", Proc. of IEEE International Symposium on Circuit and Systems (ISCAS), 1996.

[17] H. D. Chiang and M. Baran, "On the Existence and Uniqueness of Load Flow Solution for Radial Distribution Power Networks", IEEE Trans. on Circuit and Systems, Vol.37, No.3, 1990.

\section{BIOGRAPHIES}

SHIGENORI NAKA received B.S. and M.S. degrees in electrical and electronics engineering in 1992 and 1994, respectively, from Nagoya institute of technology, Aichi, Japan. He joined Kansai Electric Power Co. in 1994 and has been workin at Technical Research Center from 1999. His research interests include deregulation and distributed generation. He is a member of IEE of Japan.

TAKAMU GENJI received B.S. and M.S. degrees in electrical engineering in 1973 and 1975, respectively, from Okayama university, Okayama, Japan. He joined Kansai Electric Power Co. in 1975 and has been working at Technical Research Center from 1985. His research interests include lightning countermeasures in distribution systems and distribution automation. He is a member of IEE of Japan.

TOSHIKI YURA received B.S. and M.S. degrees in electrical engineering in 1975 and 1977, respectively, from Kyoto university, Kyoto, Japan. He joined Kansai Electric Power Co. in 1977 and has been working at Technical Research Center from 1999. His research interests include distribution systems. He is a member of IEE of Japan.

YOSHIKAZU FUKUYAMA (M'90) received B.S., M.S., and PhD degrees in electrical engineering in 1985, 1987, and 1997, respectively, from Waseda university, Tokyo, Japan. He has been working at Fuji Electric Co. R\&D Japan from 1987. He was a visiting scientist at Cornell University from 1993 to 1994 . His research interests include application of intelligent systems such as expert system, neural network, and modern heuristic techniques to power systems and power system analysis including voltage stability and load flow. He is also interested in applications of modern heuristic techniques to practical and general optimization problems. He is a member of IEEE and IEE of Japan. 